\title{
Valor de marca: un acercamiento conceptual mediante su origen y modelos
}

\author{
Brand value: a conceptual approach with its origin and models
}

Karolay Sthefany Alvarado Horna *1, Alicia Lucano Prado ${ }^{1}$
'EP. Administración, Facultad de Ciencias Empresariales, Universidad Peruana Unión

\section{INFORMACIÓN DEL ARTÍCULO}

Historia del artículo

Recibido el 11 de febrero de 2015

Aceptado el 6 de abril de 2015
Palabras clave:

Valor de marca

Percepción

Necesidades

Clientes

\section{Resumen}

La marca, en el transcurso del tiempo, ha tomado un papel significativo en el ámbito empresarial, especialmente para las micro y pequeñas empresas, ya que la incidencia de estas en el uso o imitación de marcas reconocidas, denota una clara conciencia de la importancia de la marca en el proceder comercial y en la generación de un valor agregado. Por ello, ya no se puede referir a la marca tan solo como un símbolo o imagen del producto o servicio ofertado, sino como un valor, valor que implica un grupo de fortalezas, tales como el reconocimiento de la marca, la lealtad de los clientes, la calidad percibida y asociaciones que se vinculan a esta. De aquí la importancia de conocer el verdadero significado del valor de mar$\mathrm{ca}$, por considerarse un punto crítico en las empresas, funcionando como un indicador que mide la percepción del consumidor frente a la competencia, y de permitir direccionar cada una de las estrategias y toma de decisiones, al cumplimiento de las necesidades y satisfacción de los clientes.

\begin{abstract}
Abstrac
The brand in the course of time, has taken a significant role in business, especially for micro and small businesses, since the incidence of these in the use or imitation of brands, show a clear awareness of the importance of brand in the proceed commercial, and the generation of added value. So we can not refer to the brand only as a symbol or picture of the product or service offered, but as a value, a value that represents a group of strengths, such as the recognition of the brand, customer loyalty, perceived quality and associations that are linked to this. Hence the importance of knowing the true meaning of brand value because it is considered a critical point in the business, working as an indicator that measures consumer perception against the competition, and allowing to address each one of strategies and decisions to fulfill the needs and, the customer satisfaction.
\end{abstract}

\footnotetext{
* Autor de correspondencia: Km. 19 Carretera Central, Ñaña, Lima Correo electrónico: karito12_01@hotmail.com
} 


\section{Introducción}

Una marca es un nombre, un término, un diseño, un símbolo u otra característica que identifica y diferencia a un vendedor de bienes y servicios de otros vendedores; en esencia, es la promesa de una parte vendedora de proporcionar de forma consistente a los compradores, un conjunto específico de características, beneficios y servicios. (Kotler 2002), citado por (Delgado Véliz, Patrcia, \& Víctor., 2010)

A su vez, a nivel organizacional; el valor de marca constituye uno de los principales activos intangibles de una organización, convirtiéndose en una condición necesaria para obtener resultados sostenibles en el tiempo, impidiendo caer en guerras de precio que el mercado produce y disminuyendo de forma significativa los márgenes de los competidores (Espósito, 2012) citado por (Ruiz Berger, 2012).

Bajo esta premisa, Fernandez Sabiote \& Delgado Ballester (2011) mencionan que una correcta gestión de la relación marca - consumidor se convierte en una fuente de ventaja competitiva para las empresas de hoy en día.

Por otro lado, Jean Noël Kapferer (1997) citado por (Botey López, 2010) hace referencia que la marca está considerada como el motor de la competitividad entre las empresas; ya que en la marca se sintetizan todas las funciones de una compañía, tales como, la investigación y desarrollo, producción, marketing, comunicación, etc.; todo ello para conseguir uno de sus principales objetivos que es la innovación permanente del producto y la búsqueda de una calidad cada día mayor.

De esta forma, Patiño (1995) citado por (Botey López, 2010) Ilega incluso a cuantificar sus apreciaciones asegurando que "el $36 \%$ del valor de una marca procede de la comunicación publicitaria".

Al respecto en España durante el año 2006 se anunciaron más de 90.000 marcas en los distintos medios controlados; casi un 0,8 \% más que el año anterior 2005, y de las 1,900 marcas distintas que recordaron los 11 millones de amas de casa españolas, solo 400 se situaron entre el 0,5 y el $2 \%$ de notoriedad.

A modo de ejemplo, basta indicar que de acuerdo con el informe anual editado por la agencia de medios Zenith Media (2008) la media de saturación publicitaria de las cadenas de televisión generalista en España durante el año 2007 fue de cerca del $9 \%$, es decir, de cada 100 horas de programación, 9 fueron de publicidad; esta media significa que algunas de estas cadenas de Tv llegaron a índices de saturación publicitaria cercanos al $20 \%$.

Por otro lado, el INEI (2014), realizó un estudio a base de encuestas a todas las Micro y Pequeñas Empresas, referente a cursos de capacitación relacionados con la gestión empresarial de los cuales tan solo el $2 \%$ se interesaron por el tema de marca; mostrándose de esta manera un bajo interés y, por ende, un desconocimiento de la importancia que implica tener una marca distintiva para lograr un crecimiento y un desarrollo dentro de un entorno cada vez más competitivo (Vaccaro Schmitz, 2012).

Por tanto, el presente trabajo tiene como finalidad mostrar la importancia del valor de marca, puesto que en la actualidad, es uno de los valores esenciales de la empresa; por su incidencia en las percepciones del consumidor referente al producto. Ya que una marca bien implementada se convierte en una herramienta esencial para conseguir las metas de ne- 
gocio de la empresa; esto, a su vez, permite diferenciar el producto de la competencia, garantizando su posicionamiento.

\section{Origen}

Según Costa Salá Segalés (2010), la historia de la marca y sus evoluciones son una sucesión de etapas de la praxis comercial que respondieron a situaciones y problemas cada vez más complejos. Primero pasó de la marca como signo, a la marca como garantía; luego de la marca como herramienta competitiva a la marca como valor $y$, posteriormente, de la marca como cúmulo de las etapas anteriores, a la imagen de marca.

En la primera etapa de evolución de la marca, esta fue utilizada en el antiguo Egipto, hace 2500 años como símbolo para identificar sus productos (Batey, 2013), elaborándose, según Roldán Olmedo (2010), un sistema de reconocimiento basado en tres códigos: material, formal y marcario que se hizo extensivo en Grecia y Fenicia; en tal sentido (París, 2013) hace mención que desde siempre el hombre ha usado las marcas, ya sea para reconocer a sus animales, sus posesiones y para identificarse a través de los estándares. Por otro lado, está la marca como garantía, Batey (2013) hace referencia que un nombre junto con una marca comercial es el ejemplo clásico y más rudimentario de branding, la marca como garantía de autenticidad y promesa de cumplimiento digno de confianza, es decir, la marca es la señal de autenticidad que una organización otorga como garantía de sus productos y servicios (París, 2013).

Referente a la siguiente etapa, Belén del Río, Rodolfo, \& Arguelles (2002), señalan que el término valor de la marca se comenzó a utilizar a principios de la década de los ochenta, con el fin de contrarrestar la excesiva inclinación que solían mostrar las empresas por obtener beneficios a corto plazo; de este modo, las agencias de publicidad tendieron a emplear el concepto "valor de la marca", para referirse a las ventajas competitivas que podrían obtener a mediano o largo plazo. Sin embargo, Costa Salá Segalés (2010), menciona que el valor de la marca es algo más que una simple terminología registrable basada en la originalidad, exclusividad del nombre y su representación visual, destacando que la notoriedad, confiabilidad e innovación percibida por el público, es lo que realmente genera el valor de una marca.

Por último, en la imagen de la marca, Roldán Olmedo (2010) hace mención que en el siglo XIX las marcas nacen como tales ya que, a partir del año 1950, se reconoce a la marca como una propuesta única de venta, cuya imagen ofrecía cierto grado de protección a la empresa, la cual generaba sentimientos y emociones en los consumidores. Así mismo Joan Costa (2004), citado por (Baños Gózales \& Rodríguez García, 2012), refieren a la imagen de la marca como una terminología que presenta dos acepciones: primero, como un objeto material; segundo, como una representación mental. Ambas de acuerdo a la interpretación del público, ya sea que lo relacionen con su realidad presente o con una experiencia percibida de acuerdo con lo expuesto por Kotler (2000), citado por (Baños Gózales \& Rodríguez García, 2012).

\section{Modelos de valor de marca}

\section{Modelo Brand Asset Valuator}

García Apaolaza (2012) establece que este sistema tiene en cuenta cuatro factores para determinar el valor de una marca: diferenciación, relevancia, estima y conocimiento o familiaridad. A su vez, 
Young \& Rubicam, (2009) engloba a estos cuatro factores en dos componentes: vitalidad de marca y estatura de marca, conteniendo el primer componente a los dos primeros factores presentados por el primer autor, y el segundo componente a los dos restantes.

Referente a la diferenciación, García Apaolaza (2012) menciona que es la medida en la que la marca se distingue de sus competidoras en el mercado. Es un factor vital que se ha comprobado que arrastra a todos los demás cuando empieza a declinar.

Por otro lado, Sasikala (2013) refiere que la diferenciación mide la fuerza de significado de la marca, la elección del consumidor, la esencia de la marca y el margen de potenciales; todos estos impulsados por la diferenciación, brindando a la marca la capacidad para estar al margen de sus competidores. En conclusión, ambos autores determinan que un bajo nivel de diferenciación es una clara advertencia de que una marca se está desvaneciendo.

Con respecto a la relevancia, Sasikala (2013), establece que esta mide la importancia personal de una marca en los consumidores, y está fuertemente ligado a la penetración en los hogares de los mismos. La relevancia en sí sola no es la clave del éxito de la marca, sino en su trabajo en conjunto con la diferenciación (Young \& Rubicam, 2009).

En cuanto al factor de estima, Young \& Rubicam (2009) consideran que esta mide el grado en que una marca se ve en una luz positiva y si esta se respeta. Así mismo, Sasikala (2013), proporciona un enfoque más completo, al referir que esa estima se convierte en autoestima, midiendo la calidad y las percepciones percibidas sobre el crecimiento o declive de la popularidad de una marca.
Por último, el factor conocimiento o familiaridad, Young \& Rubicam (2009), menciona que este comprueba los niveles de conocimiento y conciencia que los clientes poseen en cuestión de la marca, esto se llega a obtener por medio del verdadero conocimiento de la marca ya que si una marca ha establecido su diferenciación relevante en los consumidores y ha llegado a mantenerlo en alta estima, el conocimiento de la marca viene a representar el resultado y la culminación con éxito de la construcción de una marca (Sasikala, 2013).

En conclusión, este método supone que los cuatro factores deben de estar estrechamente interconectados, así se podrá obtener que la diferenciación multiplicada por la relevancia determine la fuerza de la marca; la estima multiplicada por el conocimiento configure la estatura de la marca, la estima define, de una mejor manera, la calidad percibida en los factores de crecimiento o decline de popularidad y por, último, el conocimiento de marca ya que el consumidor no solo es consciente de la existencia de la marca, sino que comprende lo que la marca representa y trata de transmitir (García Apaolaza, 2012).

\section{Modelo Brand Z}

De acuerdo con Kotler \& Keller, (2012) citado por (Forero Siabato, 2014), el modelo Brand Z se sustenta en la construcción de la pirámide Brand Dynamics, estableciendo que la creación de una marca se genera en la transición de etapas secuenciales tales como: vinculación, ventaja, desempeño, relevancia y presencia.

Bajo este enfoque, Kotler \& Lane, (2006), citado por (Alberto, etal, 2011), hace referencia a que el modelo Brand Z compone las características que forman la fortaleza de una marca y cuenta con 
objetivos para cada fase, en orden ascendente; por lo que las reorganiza de la siguiente forma: presencia, relevancia, resultados, ventaja y vinculación emocional.

Así mismo, Brand Z (2009), citado por (Pamplona Domínguez, 2010), hace referencia a que los resultados del presente modelo se derivan de una mezcla de datos financieros obtenidos de Millward Brown Optimor y de la base de datos WPP (que muestra resultados cuantitativos sobre las opiniones que tienen ciertos clientes sobre determinadas marcas); a partir de dicha información, se crea un proceso de evaluación que comprende las ganancias generadas por el valor de marca, cuánto de estas se deben a su acercamiento con el cliente $y$, finalmente, cuál es el crecimiento potencial de las ganancias percibidas por la marca. Además Rangel Pérez (2013), menciona que este modelo contempla la conexión entre la reputación de la empresa y la reputación de la marca, ya que, para el consumidor, se trata de un único concepto en su mente.

Con lo que refiere a la primera etapa del modelo Brand Z, Fernández Carús (2014), establece que la vinculación del consumidor con la marca, puede considerarse un factor determinante a la hora de incrementar las ventas de una empresa; por ello, el componente emocional es un aspecto de relevancia a la hora de tomar una decisión de compra, además de formar parte de las dimensiones que influyen en la construcción del valor de marca. (Kamakura \& Rusell, 1993; Keller, 1993), citado por Pérez Perna (2014).

Por otro lado, Kotler \& Keller, (2012) citado por (Forero Siabato, 2014), menciona que la fase de ventaja, corresponde a la creencia y sentimiento de que la marca ofrece una ventaja emocional y racional superior a las de la competencia.
En cuanto al desempeño, este dependerá exclusivamente de la valoración del cliente y no de la opinión de la empresa, es decir, es entendido como la percepción de que la marca ofrece un desempeño adecuado (Segarra Roca, 2007).

Por otra parte, la relevancia, de acuerdo con Rosenbaum-Elliott et al. (2011), citado por (Rangel Pérez, 2013), refleja la percepción de que la marca posee algo importante a nivel personal, es decir, apropiado para el consumidor. En tanto que en fase de presencia, donde comienza la relación del consumidor con la marca, afecta su decisión de compra, ya que una marca de fuerte presencia en el sitio de elección, hará que el consumidor tenga dudas en sus elecciones y compras. (Vásquez Valera, 2011).

Por tanto, luego de establecer el conocimiento de las cinco fases del modelo Brand Z; Kotler \& Keller (2006), menciona que los consumidores vinculados emocionalmente con la marca, es decir los que se encuentran en la cúspide de la pirámide antes explicada, desarrollan relaciones más fuertes con la marca. Sin embargo, Kotler \& Kotler (2014) establece que la mayor parte de los consumidores se encuentran en los niveles más bajo de la pirámide; por lo que el desafío de las empresas es desarrollar actividades y programas que ayuden a los consumidores a ascender en la pirámide (Kotler P. 2006), citado por Veloz Medina, (2015).

\section{Modelo de Aaker}

De acuerdo con Forero Siabato (2014), el modelo de Aaker, es uno de los pilares teóricos de la medición del Brand Equity, el cual tiene por objetivo explicar la estructura de la valoración de marca así como su gestión. A su vez, Ros (2008) 
menciona que este modelo trabaja en torno a cinco fuentes de valor de la marca, tales como la fidelidad de marca, notoriedad de marca, calidad percibida, asociaciones de la marca y otros activos en propiedad de la marca. Sin embargo, Llopis Sancho (2015) refiere que el punto fuerte del modelo de Aaker, es el amplio y detallado tratamiento que da a la definición de la identidad de marca.

Bajo esta premisa, Aaker \& Joachimsthaler (2005) menciona que la identidad de marca es aquella que establece una relación entre la marca y el cliente, generando una proposición de valor que potencialmente involucre beneficios funcionales, emocionales o de autoexpresión. Por tanto, de acuerdo a Schultz \& Jo Hatch (2010), el modelo de capital de marca presentado por Aaker, al proponer las fuentes antes mencionadas, influyen en la fortaleza de una marca y, en consecuencia, el valor que esta tendrá en el mercado.

\section{Valor de marca y dimensiones}

El valor de la marca es el valor añadido que funciona como atracción hacia un producto y/o servicio; aportando fortaleza mediante sus dimensiones, en cuanto al reconocimiento en el mercado y utilidad a sus clientes.

Bajo esta premisa, Kotler \& Keller (1998), citado por (Moreno Cabrales \& Camargo Romero, (2011), definen al valor de la marca como el valor añadido con el cual se dota a los productos y servicios; siendo utilizado como un efecto diferenciador que reconocen los consumidores, relacionado con la marca.

Por ende el valor de la marca funciona como una atracción hacia un producto en particular, generada por la parte no objetiva de la oferta del producto; no por los atributos del producto en sí. Según Lehmann (2006) citado por (Fustinoni Venturini, 2012).

Por otro lado, David Aaker (1994), citado por (Olivera García, 2012), considera que el valor de una marca constituye un grupo de fortalezas o activos como el reconocimiento de la marca, la lealtad de clientes, la calidad percibida y asociaciones; que se vinculan a la marca (su nombre y su símbolo) e incorporan (o disminuyen) valor al producto o servicio ofrecido.

Bajo la premisa anterior, Barreiro Fernández (2003) asevera que el valor de la marca es un aspecto estratégico de la dirección comercial que puede ser creado, mantenido e intensificado mediante el fortalecimiento de cada una de sus dimensiones; con el fin de conseguir un alto reconocimiento de la marca en el mercado y en sus clientes.

En conclusión, Callarisa-Fiol, Sánchez-García, Moliner-Tena y Forgas-Coll (2012), citados por (Cerdá Bertomeu, 2014), señalan que el valor de marca puede entenderse como la "utilidad global que los clientes obtienen con una marca en comparación con sus competidores".

\section{Notoriedad}

La notoriedad de marca puede conceptualizarse como una cualidad o activo intangible de la marca, relacionada con la capacidad del consumidor para recordar o reconocer la misma, y como elemento clave en la generación de valor.

Aaker et al (2000), citado por (David Chávez \& Zamora, 2014), concibe a la marca como una cualidad o activo intangible, basado en la capacidad del consumidor para recordar o reconocer la 
misma a nivel de sus signos externos; ya que estos dos últimos, forman parte de la composición de la notoriedad; siendo el reconocimiento la capacidad de un individuo para confirmar una marca determinada cuando se le ofrece entre otras, y el recuerdo la habilidad del consumidor para recordar una marca en su memoria cuando se le nombra una categoría de producto, según Keller (1993) citado por (García Rodriguez, 2009). Por ende, la notoriedad implica la presencia de la marca en la mente del consumidor, siendo una conexión aprendida entre la marca y la categoría de producto, de acuerdo a Aaker, (1994); Lambin, (1995) Samu, Krishnan y Smith, (1999), citado por (Alameda Abejón, Olarte Pascual, Reinares Lara \& Saco Vázquez, 2006)

Por otro lado, Aaker y Álvarez del Blanco (1995), citado por (Villarejo Ramos, Sánchez Franco \& Javier, 2005) mencionan que la notoriedad de la marca se conforma como elemento clave en la generación del valor de marca, debido a que esta afecta al comportamiento de compra, pudiendo incluso afectar positivamente sobre las percepciones y actitudes hacia la marca; es decir el incremento de la notoriedad incidirá de forma directa en las decisiones de compra de los consumidores como lo afirma Nedungadi (1990), citado por (Calvo Porral, Martínez Fernández, \& Juanatey Boga, 2013).

\section{Personalidad}

La personalidad de marca se puede definir en base a tres aspectos: como características y rasgos humanos que el consumidor le atribuye, como una forma de vínculo con el consumidor y como creencias que se expresan en emociones humanas.

Para Rojas Contreras \& Lara Rodríguez (2014), la personalidad de marca es el conjunto de características y rasgos de personalidad humana que el consumidor atribuye a la marca. Así mismo, Schlesinger Díaz \& Cervera Taulet (2008) mencionan que el conjunto de estas características engloban quince rasgos y cinco dimensiones: sinceridad (prácti$\mathrm{co}$, honesto, sano, alegre); emocionante (atrevido, animado, imaginativo, actualizado); competencia (confiable, inteligente, exitoso); sofisticado (clase superior, encantador) y rudeza (abierto, resistente), las cuales de acuerdo con Denegri C, Cabezas G, Herrrrera C, Páez S, \& Vargas Z (2009), se pueden medir por medio del modelo Aaker (1997), siendo este el más difundido.

Por otro lado, Ligas (2000), Fournier, (1991), citado por (Denegri C, Cabezas G, Herrrera C, Páez S, \& Vargas Z, 2009), mencionan que existe un vínculo entre los rasgos de personalidad de los individuos y los atribuidos a la marca, las cuales sirven a los consumidores como un medio simbólico para expresarse a sí mismos; es decir es el nivel de compromiso o lazos que se crean entre el consumidor y la marca, según Nobre, Becker (2010), citado por (Rojas Contreras \& Lara Rodríguez, 2014).

Por tanto, de acuerdo con Hernández \& Pérez (2009), citado por (Moreno Cabrales, Romero, \& Alberto, 2012), la personalidad de marca se funda en creencias animistas y antropomórficas; es decir, se cree que las marcas son femeninas, masculinas o ambas; que describen y expresan emociones como los humanos como lo aseveran Ben (1974); Grohmann (2009) citado por (Moreno Cabrales, Romero, \& Alberto, 2012).

\section{Calidad percibida}

La calidad percibida, puede definirse en base a dos aspectos: como el análisis 
de la calidad objetiva y la calidad percibida propiamente dicha, y como las características que el consumidor considera importante al efectuar su compra.

La calidad percibida de acuerdo con Bruno (2005), citado por Espejel Blanco \& Fandos Herrera, 2008), puede ser definida, en base a dos perspectivas diferentes: calidad objetiva y calidad percibida. En base a esta premisa, Garolera (1997), establece que la primera perspectiva se encuentra relacionada con los atributos o servicios que se incluyen en las especificaciones de fabricación; mientras que la calidad percibida, es la apreciación que tiene el consumidor acerca de la calidad global o superioridad de un producto o servicio (Zeithaml, 1988), citado por Villarejo Ramos \& Martín Velicia (2007).

Por otro lado, de acuerdo con Netemeyer (1994); y Lassar (1995), citados por Calvo Porral, Martínez Fernández, \& Juanatey Boga (2013), la calidad percibida influirá en las decisiones de compra y en la elección de una determinada marca, escogiendo aquellas en las que se percibe una mayor calidad. Por tanto, la calidad representa aquellas características que responden a las necesidades del cliente y la ausencia de deficiencias (Borré Ortiz, 2013).

\section{Lealtad}

La lealtad hacia la marca puede ser entendida como una actitud, donde se dan cabida sentimientos y afectos positivos a favor de una marca $y$; como un comportamiento conductual, materializado en compras repetidas de la misma marca.

Dick \& Basu, (1994); Oliver, (1999); Martin \& Rodríguez, (2001), citado por Colmenares D \& Saavedra T, (2007), men- ciona que la lealtad de marca está fundamentada en actitudes, mediante fases cognitivas y afectivas; expresándose por medio de la predisposición de un consumidor hacia una marca en particular, convirtiéndose de esta forma en un antecedente y predictor de la conducta de compra. (Kraus, 1995; Sample \& Warland, 1973, citado por Tobón Perilla, 2012). Sobre las bases anteriores Bloemer y Kasper, (citados en Beerli Palacio, Martín Santana, \& Fernández Moroy, 2007), establece que la lealtad es una de las formas con las que el consumidor expresa su satisfacción con el resultado del producto o servicio recibido.

Por otra parte, David Aaker (citado por Guzmán Gonzales, 2005), menciona que la lealtad de marca a menudo constituye el núcleo del valor de marca, ya que los clientes compran una marca determinada a pesar de que los competidores ofrezcan atributos superiores. En este sentido, Barrios \& Yupanqui Rodríguez (2014), establecen que la lealtad concierne al comportamiento de recompra y al compromiso que el cliente tiene con la marca; es decir, la lealtad de marca es una forma de comportamiento repetitivo de compra que se refleja en una decisión consciente de continuar comprando la misma marca (Solomon, (citado en Barrón Hernández, 2012).

Por tanto, Forgas, Moliner, Sánchez y Palau (2011) y Gómez (2010), ambos citados por Martínez Ríos (2013), definen a la lealtad, como una variable que va retroalimentándose, la cual se traduce en la retención de clientes; es decir, evitar que estos elijan otras opciones en el mercado. Tal como afirma Treviño (2006), citado por Barrón Hernández (2012), la lealtad es una acción de permanencia. 


\section{Conclusión}

La marca ha dejado de significar un simple símbolo de identificación del producto o servicio. Por el contrario, se ha convertido en el factor clave para mantener una fuerte relación con los consumidores, ya que las dimensiones del Valor de marca, tales como notoriedad, personalidad, calidad percibida y la lealtad de marca, han permitido que los consumidores se identifiquen con la empresa y se logre un afidelización con ella. Asimismo la marca se ha considerado como el motor de competitividad en una

\section{Referencias}

Aaker, D., \& Joachimsthaler, E. (2005). Liderazgo de marca. Barcelona- España: eusto.

Alameda Abejón, P., Olarte Pascual, C., Reinares Lara, E. M., \& Saco Vázquez, M. (2006). Notoriedad de marca y medios de comunicación. Revista Esic Market), mayo-agosto, (129), 91 - 116

Alberto, Z., Cuervo, S., Meza, J., Vargas, M., Velásquez, B., \& Zúñiga, M. Á. (2011). Valor de marca en el fútbol profesional.

Baños Gonzales, M., \& Rodríguez García, T. (2012). Imagen de la marca. Madrid: ESIC.

Barreiro Fernández, J. M. (2003). Gestión científica empresarial: temas de investigación actuales (Vol. 1). España: NETBIBLO.

Barrón Hernández, M. E. (2012). Factores que determinan la lealtad de marca en un producto, alimento para mascotas. (Tesis de Maestría). Universidad Autónoma de Uveretaro - México. empresa, puesto que esta se convierte en una condición necesaria para obtener resultados sostenibles en el tiempo.

Por otro lado, encontramos que entre los tres modelos para medir el Valor de marca, el modelo de Aaker permite tener un enfoque de percepción del Valor de marca en empresas medianas, lo cual facilita la inclusión del marketing en aquellas Mype que son el principal motor de trabajo del país y que, con el direccionamiento correcto, pueden lograr un fuerte impacto de estas en el cliente cada vez más exigente.

Batey, M. (2013). El significado de la la marca (Primera ed.). Argentina: Gránica.

Beerli Palacio, A., Martín Santana, J., \& Fernández Moroy, M. (2007). Importancia del valor de marca en la satisfacción y lealtad de los clientes en el mercado industrial.

Del Río Lanza, A.B., Vásquez Casiellas, R., \& Iglesias Arguelles, V (2002). El valor de marca: perspectivas de análisis y criterios de estimación. Cuadernos de Gestión.

Borré Ortiz, Y. M. (2013). Calidad percibida de la atención de Enfermería por pacientes hospitalizados en Institución Prestadora de Servicios de Salud de Barranquilla.

Botey López, J. (2010). Aproximación teórico-empírica a la figura del director de cuentas como el gestor de la comunicacion de marcas en agencias de publicidad de Barcelona. Barcelona: Universitat Ramon Lull Fundació Privada.

Bravo Gil, R., Fraj Andrés, E., \& Martínez Salina, E. (20 de Abril de 2006). La influencia de 
las fuentes de información externas en el valor de marca: una perspectiva desde el joven consumidor. Cuadernos de Estudios Empresariales, 16, 9-32.

Buil, I., Martínez, E., \& De Chernatony, L. (5 de Marzo de 2010). Medición del valor de marca desde un enfoque formativo. Universidad de Zaragoza .Cuadernos de Gestión, 10(Especial), 167-196.

Calvo Porral, C., Martínez Fernández, V. A., \& Juanatey Boga, O. (octubre de 2013) Análisis de dos modelos de ecuaciones estructurales alternativos para medir la intención de compra. Revista Investigación Operacional. 34(3), 230 - 243.

Cerdá Bertomeu, M. J. (2014). El papel de las Administraciones Públicas al crear Marcas Territorio: Una perspectiva de marketing. (Tesis doctoral). Universidad Miguel Hernández, España.

Colmenares, O. A., \& Saavedra T, J. (2007). Aproximacion teórica de la lealtad de marca: enfoques y valoraciones. Cuadernos de Gestión. 7(2), p 69-81

Coronado Barrios, F. J., \& Yupanqui Rodríguez, D. G. (2014). Posisionamiento de marcas de calzado para mujeres entre 20-26 años en la cuidad de chiclayo. (Tesis de licenciatura). Universidad Católica Santo Toribio de Mogrovejo. Perú

Costa Salá Segalés, J. (2010). La marca: creación, diseño y gestión. México: Trillas, S.A. de C.V.

Fisher L., Chávez, D. \& Zamora, O. (2014). La relación entre el valor de la marca percepcion de marca e imagenes afectivas IAPS (International Affective Picture Sistem). European Scientific Journal, 10(10), p. 66-79

De la Fuente Mella, H., \& Rey Gaete, F. (2009).
Análisis de la calidad percibida, imagen corporativa, satisfaccion y lealtad de los clientes de los supermercados: Una aplicación para un supermefrcado de Chile. (Proyecto Social: Revista de Relaciones Laborales, 12(12), p. 77-102)

Delgado Véliz, J. R., Patrcia, P. R., \& Víctor., R. M. (2010). Estudio sobre dimensiones de la personalidad de la marca en Perú. Perú: Pearson .

Denegri C, M., Cabezas G, D., Herrera C, V., Páez S, A., \& Vargas Z, M. (2009). Personalidad de marca de carreras de psicología de universidades estatales en Chile: un estudio descriptivo. Revista de Investigación en Psicología, 12(2).

Espejel Blanco, J., \& Fandos Herrera, C. (2008). La calidad percibida como antecedente de la intención de compra del aceite de oliva del bajo Aragón con denominación de origen protegida.

Fernández Carús, L. (2014). El comportamiento del consumidor Online: Factores que aumentan la actividad de búsqueda de EWOM en el sector turístico. España: Universidad de Oviedo.

Fernández Sabiote, E., \& Delgado Ballester, M. E. (2011). Marcas de experiencia marcando la diferencia. Estudios Gerenciales.

Forero Siabato, M. F. (2014). Determinación de la aplicabilidad del Brand Equity basado en el consumidor para el contexto colombiano. Bogotá- Colombia: Universidad Nacional de Colombia.

Francisco, V. R., Sanchez Franco, M. J., \& Rondán Cataluña, F. J. (2008). Antecendentes de la notoriedad del nombre en la determinación en la imagen de marca. Una visión desde un producto de gran consumo. Universidad de Sevilla. 
Fustinoni Venturini, M. (2012). Cartera de marcas de distribuidor en el establecimiento minorista: Universidad de Salamanca. España: Departamento de Administración y Economía de la Empresa.

García Apaolaza, A. (2012). La gestión de la marca en el sector hotelero. Oviedo: Universidad de Oviedo. Facultad de economía y empresa.

García Rodriguez, M. J. (2009). El papel de la notoriedad de marca en las decisiones del consumidor.

Garolera, J. (1997). Aspectos distintivos del "Brand Equity" en marcas percibidas como locales y como globales.

Gúzman Gonzáles, M. J. (2005). El valor de la marca y su efecto en la lealtad y la fijación del precio por el consumidor. Ecuador: Escuela superior politecnica del litoral.

INEI. (2014). Resultados de la Encuesta de micro y pequeña empresa. Lima.

Kotler, P., \& Keller, K. L. (2006). Dirección de marketing. México: Pearson educación.

Kotler, P., \& Kotler, M. (2014). 8 Maneras de crecer. Madrid- España: LID.

Llopis Sancho, E. (2015). Crear la marca global: modelo práctico de creación e internacionalización de marcas. MadridEspaña: ESIC.

Martínez Ríos, O. (2013). Lealtad de marca hacia una empresa aseguradora, bajo la perpectiva sociodemografica de su mercado actual en tijuana, B.c.

Moreno Cabrales, F. A., \& Camargo Romero, L. A. (2011). Teorías constitutivas de la marca. Colombia: Colegio de estudios superiores de administración.
Olivera García, J. L. (2012). Valoración de la marca. Revista de Derecho de la Universidad de Montevideo.

Pamplona Domínguez, M. (2010). El reinado de las marcas. Monterrey- Mexico: Tecnológico de Monterrey.

París, J. A. (2013). La marca y sus significados (Primera ed.). Madrid: Ediciones Haber.

Pérez Perna, A. (2014). Gestión estratégica de la marca coorporativa en los mercadis financieros. Referencia al grupo SANTANDER. España: Univerdidad de Coruña.

Rangel Pérez, C. (2013). La nueva gestíon de las marcas: una visión en el sector de lujo. Madrid. : Universidad de complutense de Madrid. .

Rojas Contreras, C. A., \& Lara Rodríguez, J. S. (2014). Relacion entre involucramiento y personalidad de la marac, una revisión.

Roldán Olmedo, S. (2010). Brand Equity. El valor de la marca en tiempo de crisis económica. Argentina: Universidad de Palermo. Facultad de Comunicación y Diseño.

Ros, V. (2008). Posiciona tu marca en la red: e- Branding. Madrid- España: Netbiblo. S.L.

Ruiz Berger, J. P. (2012). Propuesta de modelo de medición de imagen de marca. Valparaíso: Universidad Técnica Federico Santa María .

Sasikala, D. (2013). Brand Asset Valuator- measuring brand value. International journal of social science \& interdiciplinary research, 2.

Schlesinger Díaz, M. W., \& Cervera Taulet, A. (2008). Estudio comparativo entre per- 
sonalidad de marca ideal vs. percibida: aplicación a las compañías aéreas.

Schultz, M., \& Jo Hatch, M. (2010). Esencia de marca. España: LID.

Segarra Roca, P. (2007). Influencia de la heterogeneidad del mercado en la intención de comportamiento del consumidor: Respuestas a la actividad relacional en la distribución de gran consumo. Cataluña- España: Universitat Rovira I Virgili.

Tobón Perilla, S. M. (2012). Lealtad conductual con discriminación marca/producto entre consumidores con historia diferencial de consumo. Bogotá: Universidad Nacional de Colombia.

Vaccaro Schmitz, C. (2012). Distintividad y uso de las marcas comerciales. Derecho Económico.

Vásquez Valera, M. P. (2011). El proceso de construcción de marca en las instituciones de educación superior Universidades de Manizales. Colombia: Universidad Nacional de Colombia.

Veloz Medina, J. M. (2015). La gestión de la marca y su posicionamiento en el mercado para la empresa productora de harinas MAGIVEL de la ciudad de Ambato. Ecuador: Universidad Ténica de Ambato.
Vera Martínez, J. (2015). Perfil de valor de marca y la medición de sus componentes. Revista Latinoamericana de Administración.

Villarejo Ramos, A. (2009). Modelos multidimencionales para la medición del valor de marca. Universidad de Sevilla.

Villarejo Ramos, Á. F., \& Martín Velicia, F. A. (2007). Una propuesta de modelo para la medición del valor de marca en entidades deportivas.

Villarejo Ramos, Á. F., Sánchez Franco, M. J., \& Javier., R. C. (2005). Antecedentes de la notoriedad del nombre en la determinacíon de la imagen de marca. Una visión desde un producto de gran consumo.

Ydrogo Rojas, C. K. (2015). Análisis de valor de marca para zapatillas deportivas, en jóvenes de 18 a 25 años de la ciudad de Chiclayo. Chiclayo: Universidad Católica Santo Toribio de Mongrovejo.

Young, \& Rubicam. (2009). Brand Asset Valuator power grid. Europan institute for brand managament. 